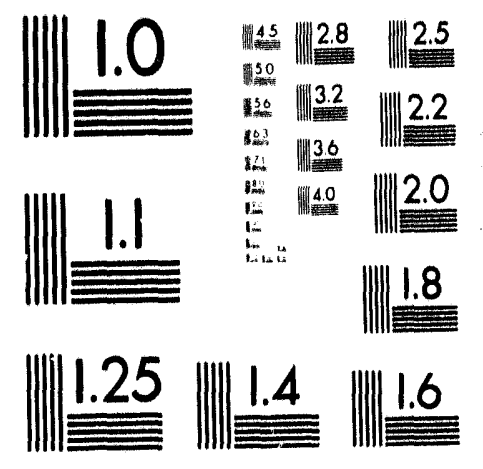




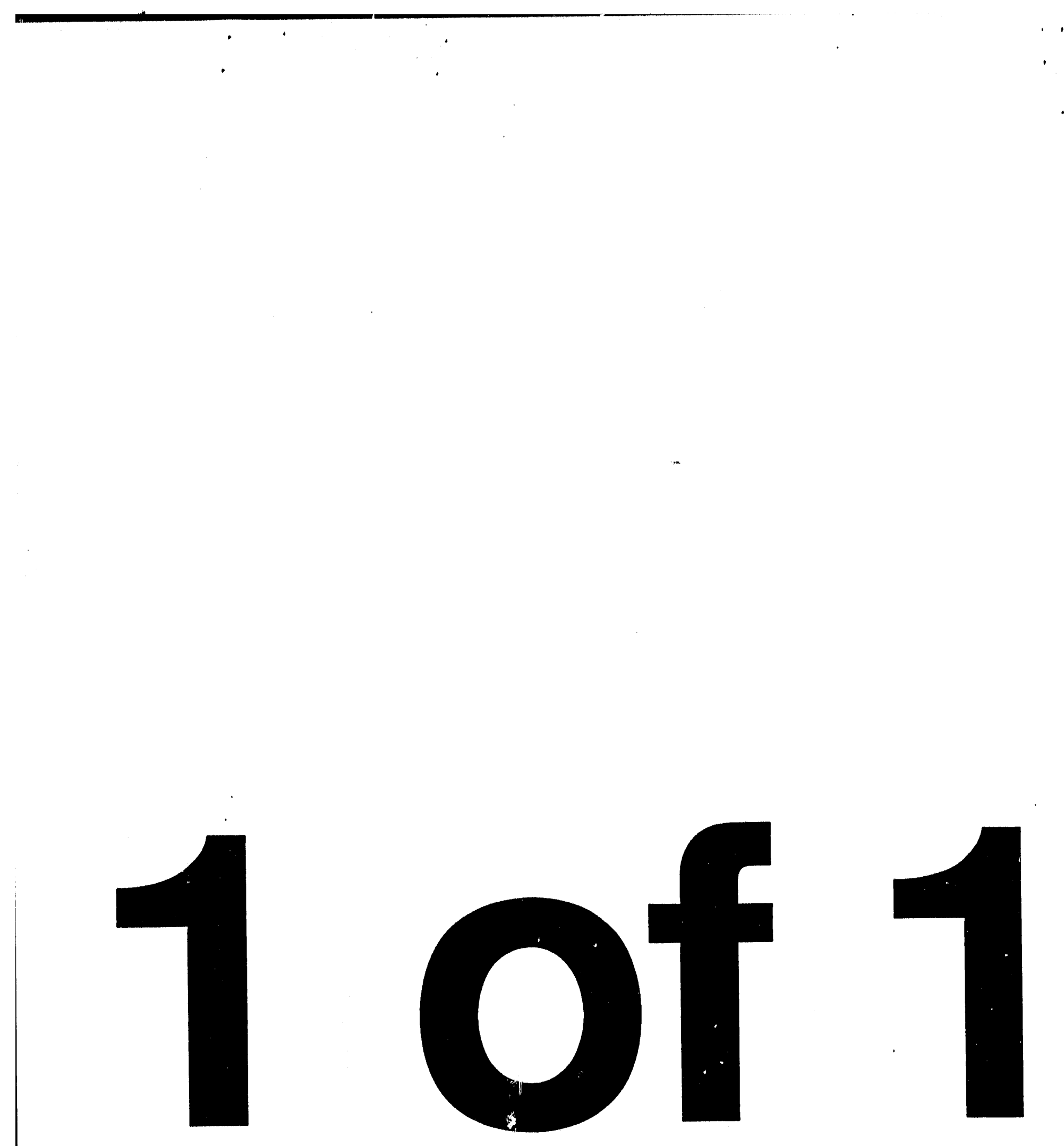





\title{
The Overview and History of Permanent Magnet Devices in Accelerator Technology*
}

\author{
Robert H. Kraus Jr. \\ Los Alamos National Laboratory \\ Los Alamos, NM 87545
}

\begin{abstract}
This paper reviews the early history of accelerator development with a partlcular focus on the Important discoverles that opened the door for the application of permanent-magnet materials to this area of sclence. Researchers began to use permanent-magnet materials in particle accelerators soon after the Invention of the alternating gradient principle, that showed magnetic flelds could be used to control the transverse envelope of charged. particle beams. Since that tlme, permanent-magnet materials have found wide application in the modern charged partlcle accelerator. The history of permanent-magnet use in accelerator physics and techuology is outlined, general design considerations are presented, and material propertles of concern for partlcle accelerator appilcations are discussed.
\end{abstract}

\section{INTRODUCTION}

The lodestone (the mineral magnetite) was the first permanent-magnet (PM) or permanently magnetized material known to man and predated the first charged-particle accelerator by at least two millennia. The earliest recorded observation of permanent magnetism in magnetite occurred in ancient Greece between 200 and 100 BCE. The electromagnet was not invented until 1825, when William Sturgeon presented to the members of the Society of Arts in England his "horse-shoe" magnet wound with 18 turns of copper wire through which a current was passed. The discovery of electromagnets that could produce flux densities far greater than the permanent magnets available at that time generated enormous excitement and may have caused permanent magnets to fade into obscurity for many years.

In 1919 Rutherford[1] transmuted nitrogen nuclei using "radiations" (alpha particles which are helium nuclei stripped of both electrons) from naturally occurring thorium sources. These experiments were part of a new frontier of science being explored early in this century that has developed into the nuclear physics and nuclear chemistry of today. Rutherford recognized the limitations of natural sources of energetic particles and, in 1927, advocated the construction of particle accelerators that could accelerate charged nuclear particles to sufficiently high kinetic energy to transmute or disintegrate nuclei[2]. Thus began the history of particle accelerators.

During the last 50 years, the particle accelerator has grown from a research instrument unique to nuclear science to become an important tool in a wide variety of scientific, medical, industrial, and defense fields including nuclear and

\footnotetext{
* Work supported by the Los Alamos National Laboratory Institutional Supporting Research, under the auspices of the US Department of Energy.
}

particle physics, material science, astrophysics, medical therapy, radioisotope production, the semiconductor industry, the food industry, and the Strategic Defense Initiative. Although PM materials were not immediately seized upon for use in particle accelerators, as the field of accelcrator science matured, the role of permanent magnets has grown and will likely continue to grow in importance. This paper presents how PM technology has impacted the development of accelerator science and engineering and what might be anticipated in the future. Permanent-magnet technology has had a profound impact on insertion devices (wigglers and undulators), but this topic is not covered in this paper.

\section{AN HISTORICAL OVER VIEW}

Before Rutherford's speech to the Royal Society[2] advocating the development of accelerators for use in nuclear physics, Ising [3] proposed a concept in 1924 whereby charged nuclear particles could be accelerated by repeated application of an electric field. Wideroc, in his famous 1928 paper [4], published the first successful account of accelerating positive ions in a structure composed of a series of drift lubes, a "linac". Shortly after World War II, Luis Alvarez $[5,6]$ led a group at the University of Califormia Radiation Laboratory at Berkeley that designed and built the first linac capable of producing beams of protons with sufficient energy for nuclear physics experiments. The accelerator designed by Alvarez was the forenunner of modem "drift-tube linac" that bears his name. Alvarez and others recognized the need for transverse focusing to offset the net defocusing effect of the ions traversing the electric field between drift tubes. Metal foils were initially placed over the entrance aperture of the drift tubes to modify the electric field; however, scattering caused $~ 50 \%$ beam loss.

After the historic paper by Courant, Livingston, and Snyder[7] describing the alternating-gradient (AG or strongfocusing) principle, magnetic fields began to play an important role in controlling the transverse envelope of charged-particle beams. Blewett[8] described the strong. focusing principle as it specifically applied to the linac. Alvarez and coworkers $[6,9]$ reported beam intensities increased by a factor of two in the first test of the AG principle on the newly constructed $32 \mathrm{MeV}$ proton linac at Berkeley. Several reports soon appeared in the literature describing magnetic strong-focusing devices that were used in the transport of charged particle beams outside an accelerating structure [1012,16]. After the first installations of magnetic strongfocusing in accelerator systems in 1959 [13,14], virtually every new accelerator incorporated magnetic strong-focusing in the accelerator structures[15].

Clogston and Heffner[16] first proposed using PM materials to supply the magnetic flux for a strong-focusing system in 1954. At the University of California Radiation 


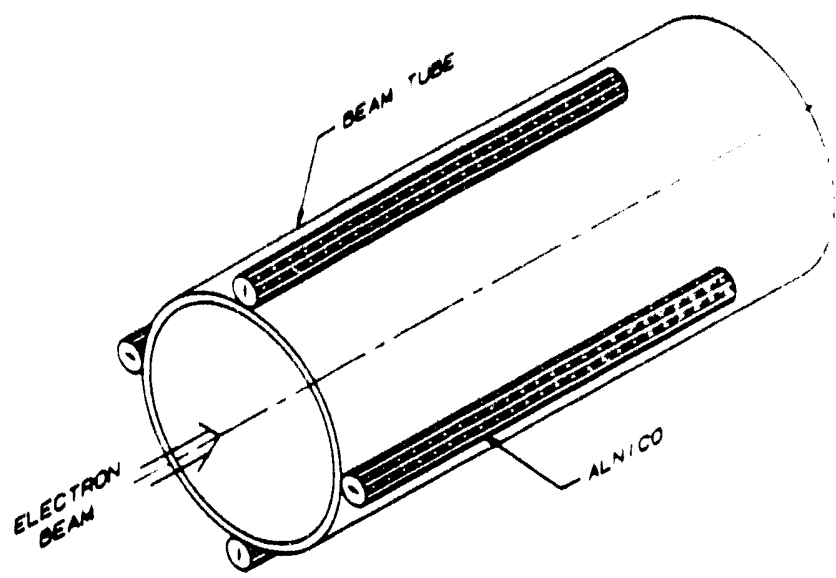

Fig. 1. An illustration of the placement of cylindrical Alnico blocks around the beam tube by $W$. Stubbins $[17]$ to form a quadrupolar field. The easy-axis orientation of the permanent magnet bars is indicated by arrows drawn inside the cylinders.

Lab. Stubbins[17] applied the concept in 1955 to control the divergence of a $7 \mathrm{MeV}$ electron beam through a transport line. He arranged four cylindrical Alnico bar magnets at $90^{\circ}$ increments around the bean tube as illustrated in Fig. 1 , producing a focusing field inside the beam pipe. An optimum configuration for rontrolling the beam divergence was found experimentally in this first lest of the strong-focusing principle using permanent magnets. Stubbins also exhibited great foresight in suggesting that PM focusing devices could be ... in "confined regions such as drift tubes." In 1955. Wucox [18] proposed the first Cockcroft-Walton injector with PM quadrupoles, and Keller[19] developed simple pernnanentmagnet quadrupoles (PMQs) in 1960 for a polarized ion source. All of these authors argued that increased simplicity, lack of power supplies, and no cooling requirements justified developing PM devicis to replace traditional electromagnets.

Jagger ano Riley[20], in 1967, designed and fabricated

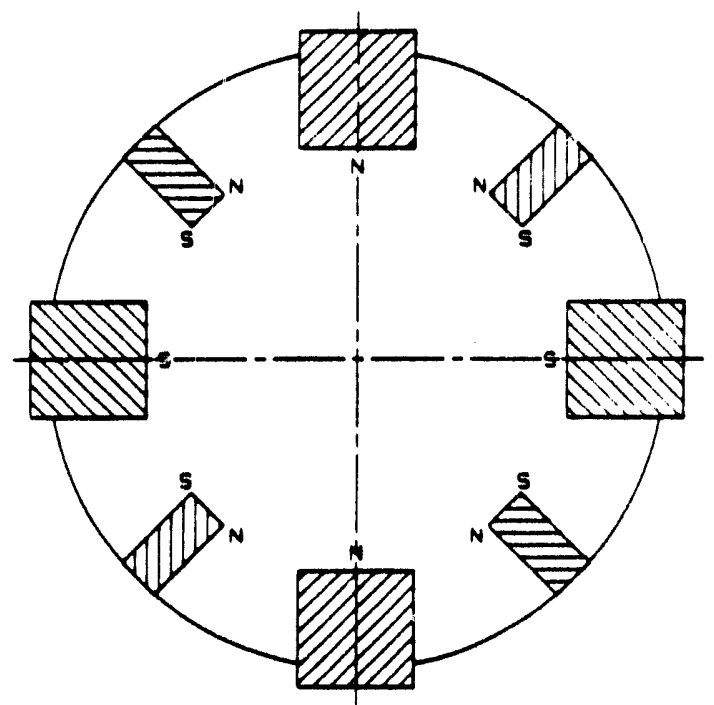

Fig. 2. The placemeni of barium ferrite PM blocks on an iron yoke to form a quadrupolar field by Jagger and Riley[20] in 1967 is shown. The orientation of the easy axis of the PM bars is indicated by the arrows drawn inside the blocks. quadrupole magnets, schematically shown in Fig. 2, that used PM pole blocks mounted on an external soft-iron flux-retum yoke. The smaller permanent magnets placed between the primary pole blocks were used to improve the quality of the field and increase the magnetic field gradient to 4.7 Tesla/meter $(T / \mathrm{m})$ for an $8 \mathrm{~cm}$ clear aperture (effective pole-tip flux density of $0.18 \mathrm{~T}$ ). The authors also described a scheme to vary the focusing strength (gradient) of single magnets from $0 \mathrm{~T} / \mathrm{m}$ to $4.7 \mathrm{~T} / \mathrm{m}$ by moving them longitudinally over a mild steel shunt, however, the mechanism required a relatively large space along the beam line (a luxury not often available).

In 1968, Harvey and coworkers[21] published an account of their use of PM focusing in the linac at the Chalk River National Laboratory (CRNL). Their design used barium ferrite to supply the magnetic flux and an iron return yoke. Harvey, et al. determined that the field quality required to reduce beam spill to an acceptable level necessitated the use of iron poles to precisely shape the field. Permanent magnets were used only to supply the magnetic flux for an iron core, very similar to an electromagnet, often called a "coil replacement" design. The magnets built at CRNL produced field gradients up to $9 \mathrm{~T} / \mathrm{m}$ with a $5 \mathrm{~cm}$ aperture, resulting in an effective pole-tip flux density of $0.23 \mathrm{~T}$.

Interest in permanent magnet devices that had waned in the late 1960's and early 1970's was rekindled in the mid 1970's when several new papers appeared describing a variety of designs and applications. The first after the hiatus described the simple application of permanent magnets to eliminate $X$ ray and $\gamma$-ray backgrounds from electrostatic-accelerating tubes and cavities[22,23]. Jim Bradbury[24], at the Los Alamos National Laboratory (LANL), recognized in 1975 that permanent magnets would be an integral part of a new class of linacs being developed for medical applications that needed to exhibit lower cost, compactness, simplicity, and high reliability. Bradbury asserted that increasing the accelerator frequency was necessary to attain these goals. Electromagnets for such applications would become increasingly difficult to fabricate, operate, and maintain; and, above $-400 \mathrm{MHz}$ it is no longer technologically feasible to build them small enough to fit in the drift tubes. Bradbury's paper presented the first organized rationale for using permanent magnets in accelerators. A flood of papers appeared in the literature soon after 1975 that exploited various aspects of PM materials for accelerator applications [22-33].

Figure 3 shows the flux density at the effective pole-tip (Bpole) of quadrupole magnets plotted as a function of the year the work was published for a sampling of quadrupole magnets that have been built and tested. Two features worth noting in this historical perspective of PMQ development are the monotonic increase of Bpole for nearly 25 years and the impact the material type has had on the performance. Alnico has a practical maximum $B_{\text {pole }}$ of approximately $0.2 \mathrm{~T}$, ferrites can produce $0.5-0.6 \mathrm{~T}$, and rare earth materials nearly $1.3 \mathrm{~T}$. It is apparent that the performance of PMQs over the years is strongly coupled to material advances.

Use of REC materials for accelerator magnets was proposed in 1977 by Mishin and Grechishkin[28]. The first magnets fabricated with REC materials and iron poles to concentrate and shape the field were reported in 1977 by Swenson, et al.[30] for a quadrupole design and by Ilyushchenko and Kilikov[31] for a dipole design. Saito, Bush, and 


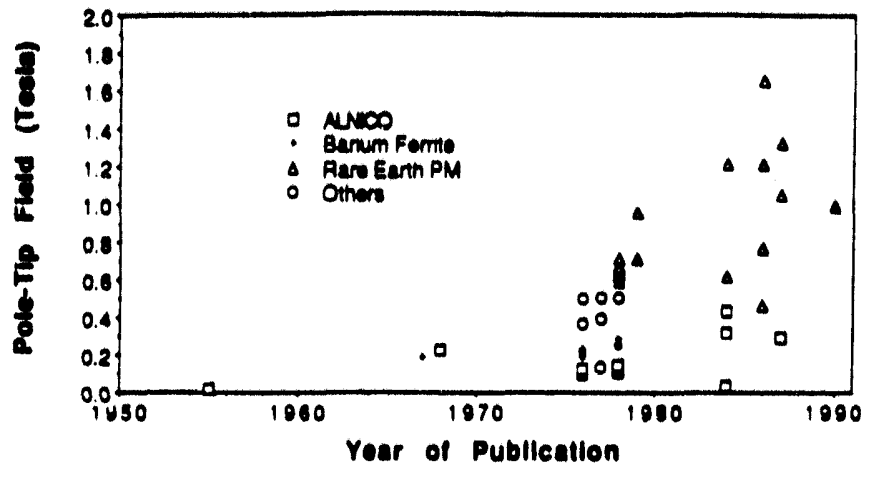

Fig. 3. A plot of the effective pole-tip field as a function of the year the work was published for quadrupole magnets fabricated with various permanent-magnet materials. The "Rare Earth PM" designation represents data for magnets fabricated with either Sm-Co or Nd-Fe-B materials.

Swenson[32] published a comparative study of the performance of several prototype quadrupole magnets using different PM materiais and d'fferent design concepts. Saito and collaborators reported effective Bpole over $0.67 \mathrm{~T}$ and gradients over $100 \mathrm{~T} / \mathrm{m}$ for magnets using SmCos materials. The highest Bpole obtained for Alnico-V and barium ferrite materials for these same designs were $0.15 \mathrm{~T}$ and $0.29 \mathrm{~T}$, respectively. REC materials not only have significantly larger magnetic remanence than the ferrites but also have the property that the slope of the B-H curve is very nearly 1.0. This is equivalent 10 saying that the permeability of the material is nearly 1.0, thus the material will superpose with lines of magnetic flux without perturbing those lines. The realization of this property opened the door for new applications such as mounting a REC magnet inside the magnetic field of a colliding-beam detector to increase the luminosity at the interaction region[34].

A pivotal design concept based on $\mu=1.0 \mathrm{PM}$ material was implicitly proposed by Blewett [35] in 1965 and independently described in practical terms by Lazarev and Skachkov[33], Halbach[36], and Holsinger[37] in 1979 for oriented PM materials. The magnet fabricated by Holsinger consisted of trapezoidal segments arranged into a cylinder as shown in Fig. 4. This geometry packs the maximum REC material into a
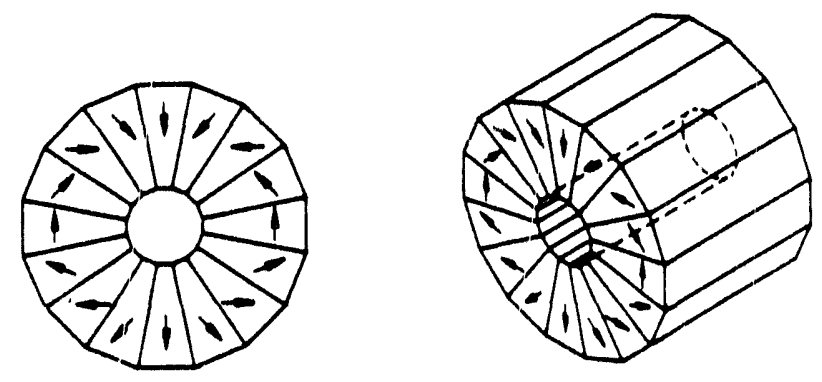

Fig. 4. A schematic diagram of a segmented magnet as proposed by Halbach and Holsinger[36, 37]. The example shown here generates a dipole field distribution in the apenture. but other multipoles can be produced[36]. given volume, however, the segments must be ground to high precision and are "locked" in place. Lazarev and Skachkor fabricated a magnet of cylindrical SmCos blocks mounted in a non-magnetic ring as shown in Fig. 5. This design generates lower fields than the previous design; however, precision grinding of PM blocks is not required and the design can be "tuned" by fine rotations of the PM cylinders. Lazarev and Skachkov reported measuring an Bpole of $0.71 \mathrm{~T}$ for their prototype, while Holsinger reported $0.96 \mathrm{~T}$.

Skachkov and co-workers[38] have very recently proposed and successfully tested a technological innovation for producing permanent-magnet multipoles with larger effective pole-tip fields. Rather than constructing a cylindrical magnet using discreet blocks of permanent magnets they fabricated a single cylinder of PM material with a continuously varying magnetization vector. This design, with the $100 \%$ fill-factor of PM material and the continuously rotating magnetization vector attains the theoretical limit of the aperture field as originally proposed by Blewetu[35].

Variability of the magnetic moment is of ten required for devices used in accelerator applications. Holsinger[37] proposed that multiple magnets placed longitudinally along the beam pipe could be rotated relative to one another to vary the integrated gradient. This design generates high flux densities, but couples a rotation of the 2-D quadrupolemoment (phase) with the variation of the quadrupole moment, adversely affecting the beam. Meanwhile Lazarev and Skachkov[33] suggested that two concentric magnets around the beam pipe could be moved in a counter-rotating fashion to vary the field. The 2-D quadrupole phases from the inner and outer magnets cancel resulting in no coupling of the net phase with the variation in amplitude. Precision ion-optical calculations must, however, take the fringe fields into consideration for which cancellation of the phase rotation is not perfect.

Lazarev and Skachkov fabricated a prototype of the variable-field permanent-magnet (VFPM) design proposed in their paper showing that any field gradient between $0 \mathrm{~T} / \mathrm{m}$ and the maximum value of $62 \mathrm{~T} / \mathrm{m}$ could be generated. In two later papers, Gluckstern and Holsinger[39,40] presented

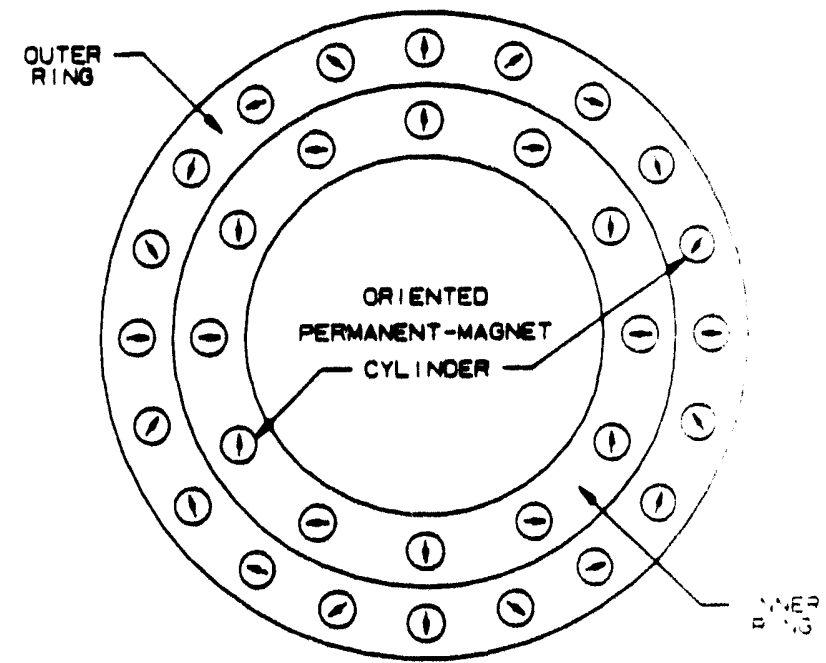

Fig. 5. A schematic diagram of a "tipless" REC permanent magnet as proposed by Lazarev and Skachkov. The specific example shown here generates a quadrupole field distribution in the aperture, but other multipoles can be produced [33]. 
methods for cancelling the effects of the phase rotation of the original design(37) using multiple disks (a five-disk singlet) to vary the strength of the lens. Zeller and coworkers[41] constructed a group of rotating VFPM quadrupoles for application in the National Superconducting Cyclotron Laboratory (NSCL) K800 cyclotron transport line. The application required resistance to a high radiation flux, a small radial envelope, and absence of highly permeable materials.

In 1983, Halbach proposed a novel design for a VFPM quadrupole that does not couple phase rotation with variability in 2-D, is compact, and can be constructed with pole-tip flux densities up to $\sim 1.4 \mathrm{~T}$. A schematic and detailed description are shown elsewhere in these proceedings[42] and in ref. 43. The inner core of this magnet is a coil-replacement design consisting of four iron pole pieces and four PM blocks supplying magnetic flux. The outer ring consists of an iron yoke and four sections of PM material. Rotating the outer ring assembly with respect to the inner core, that remains fixed in the laboratory frame, varies the flux density in the poles and consequently in the magnet aperture and not the orientation (phase) of the 2-D field. Measurement data for magnets fabricated at LANL for the Superconducting Supercollider (SSC) are presented elsewhere in these proceedings[42]. The first prototype magnet of this type was built in 1985 by Halbach and coworkers[44].

The "laced"-magnet is yet another design proposed by Halbach[45] in 1986 that is essentially identical to iron-core electromagnets, but can generate higher effective pole-tip flux densities by using permanent magnets to reduce flux loss in the iron. The permanent magnets produce a $B$ in the iron generally oriented in the opposite direction to the B generated by the coils. The total field in the iron is reduced, consequently reducing saturation effects and associated losses. Feinberg and coworkers[46] were the first to fabricate a prototype of the laced magnet and later units were constructed and installed into the SuperHIL AC at LBL.

Another variable-field concept, presented in detail elsewhere in these proceedings, is a VFPM dipole designed at LANL that uses a specially shaped movable shunt to vary flux density in the gap in a nearly linear fashion while reducing the force on the shunt[47]. These magnets, along with VFPM quadrupole doublets, were used in the Advanced Free-Electron Laser (AFEL) program at Los Alamos[47]. The highest beam brightness ever recorded for an FEL was observed at AFEL, owing in part to the high quality of the VFPM devices.

Today, PM materials are used throughout accelerators from ion sources to output optics. Numerous other applications include the useing PM meterials for ion sources, in particular the ECR (electron cyclotron-resonance) ion source $[48,49]$. Geller[49] points out that an ECR source with a PM ionconfinement field consumes no power and performs as well as earlier ECR sources that consumed $3 \mathrm{MW}$ of power. Ion sources and low-energy transpont lines use solenoidal fields for which PM solutions have been suggested[50,51]. Permanentmagnet materials have also been used as integral parts of devices peripheral to the accelerator proper such as in highpower Klystrons[52], and a variety of detectors [53,54].

The GTA accelerator[56] also uses permanent magnets in two other novel applications. The drift-tube linac is designed to operate at cryogenic (non-superconducting) temperatures requiring the $\mathrm{PM}$ drift-tube quadrupoles to operate at $20 \mathrm{~K}[57,58]$. A second unique feacure of the GTA accelerator is the $1.25 \mathrm{~m}$ aperture PM output optics used to produce a very parallel, high quality beam, analogous to an optical telescope operating in reverse. Combined-function quadrupole and octupole magnetic lenses are used to expand the beam and to correct the third-order geometric aberrations induced in the beam[59] by the focusing action. Correction of beam. aberrations using permanent magnets was also implemented at the Stanford Linear Accelerator Cenier $[60,61]$.

PM devices are being considered for final-focus optics in the next generation of very high-energy colliding beam accelerators or heavy-ion induced fusion systems. Extraordinarily large focusing strength and small apertures will be required, complicated by the likely location in or near strong magnetic fields from the interaction-region detector. Studies indicate that linear field gradients in excess of 1000 $\mathrm{T} / \mathrm{m}$ can be generated by pure PM and hybrid PM/iron designs using existing materials [62-64].

In the last decade, a new group of PM materials, rare earthiron compounds, have been developed. $\mathrm{Nd}-\mathrm{Fe}-\mathrm{B}\left(\mathrm{Nd}_{2} \mathrm{Fe}_{1,4} \mathrm{~B}\right)$ has a remanence and intrinsic coercivity $10-30 \%$ greater than the rare earth-cobalt compounds[55]. Studies of this material for accelerator applications have been reported by several authors $[60,64,65]$ and prototype magnets have been fabricated $[60,66,67]$. The highest value plotted in fig. 3 represents data reported by Kumada and coworkers[66] for a magnet constructed using the Nd-Fe-B. Nd-Fe-B has the adverse properties of large thermal coefficients of magnetization and greater susceptibility to damage by radiation than $\mathrm{Sm}-\mathrm{Co}$ compounds. Our group is currently testing a new material, Pr2 Fe 14B, developed by Sankar and associates [68] for potential accelerator applications. This material is important because the remanence is some what larger than $\mathrm{Nd}-\mathrm{Fe}-\mathrm{B}$, it has a higher Curie temperature, $\mathrm{T}_{\mathrm{C}}$. (resulting in a lower thermal coefficient of magnetism), and exhibits no spin reorientation down to $4 \mathrm{~K}[69]$.

\section{GENERAL DESIGN CONSIDERATIONS}

The design details for most of the magnets discussed in the preceding section are presented in the cited literature, in ref. 70, and refs. therein. This section outlines some general considerations that often impact PM designs for accelerator applications.

Meeting magnetic field strength and quality requirements are the primary goals when designing magnets for accelerator applications. The range of variability and how precisely the magnetic field can be set are also important for variable-field designs. A large variety of codes for magnet modeling and design are available Loday; those commonly used at Los Alamos include PANDIRA[71], FLUX[72], and TOSCA[73]. Although it is common to design magnets assuming ideal materials (i.e., linear B-H behavior for PM materials, homogenous materials and material properties, perfectly oriented magnetization vectors, etc.), modeling codes can be used to simulate "real world" materials and tolerances. Modeling imperfections tends to be tedious, and is typically used to formulate mechanical and magnetic material tolerances. Ultimately, the quality of a magnet is determined by measurement. Magnets using only $\mu=1.0 \mathrm{PM}$ materials can usually be designed well with analytic formulae $[36,37]$. 
The trade-offs between segmented[36,37] (only $\mu=1.0$ materials) multipole magnets and those using highly permeable materials must be considered in the design process. Segmented multipole magnets are generally more efficient and easier to design than iron-core magnets. Analytic design formulae may, however, mislead a magnet designer into believing that field quality for a typical segmented multipole magnet is as good or better than that obtainable with iron-core designs. Theoretically, the first-allowed harmonic in an ironcore quadrupole magnet is $n=6$, while for a 16-segment pure $P M$ magnet the first-allowed harmonic is $n=18$. However, variation in material properties and manufacturing tolerances invariably produce lower-order disallowed harmonics[70]. In our experience at Los Alamos, it has been more difficult to obtain PM material with near-ideal properties than to obtain near-ideal iron. Consequently, when the quality of the magnetic field is the highest priority for a magnet design, one should focus on an iron-pole design when possible. Iron will homogenize small imperfections in the PM material while shaping the aperture field to the desired profile. Techniques for reducing error harmonics in segmented magnets, though tedious and labor intensive, have been developed[70].

The thermal environment during fabrication and operation is an important general consideration for the magnet designer. Many PM materials are sensitive to extreme temperatures often requiring special fabrication procedures to prevent irreversible damage. Permanent-magnet quadrupole (PMQ) magnets are welded into GTA drift tubes using an electronbeam tochnique that maintains the magnet temperature below $100^{\circ} \mathrm{C}$. The operational temperature and any thermal gradients expected during operation may impact the design of magnets using PM materials. REC and Nd-Fe-B materials have very large thermal coefficients of remanence; hence, changes in ambient temperature alter the field strength and thermal gradients may impair the field quality of PM devices. These properties are discussed in section 4.

Finally, it is crucial that the radiation environment to which the PM material will be subjected is taken into consideration when designing a magnet. Radiation, especially nuclear particles such as neutrons and protons, cause both reversible and irreversible damage that may limit the operational lifetime of PM devices. A detailed discussion of the effects of several types of radiation on a variety of PM materials is presented in the following section. Careful attention should be given to this discussion and to the cited literature before determining the suitability and choice of material for permanent magnets in high-radiation applications.

\section{PERMANENT-MAGNET MATERIAL PROPERTIES}

The material properties of permanent magnets play an important role in determining the suitability of these materials for application in accelerators. B-H properties, remanence, intrinsic ccercivity, energy product, and permeability all affect the design characteristics and performance of a magnet through a set of interrelations too extensive to delve into in this review paper. The reader is referred to refs. $39,40,70,74,75$ to begin a study of the effects of these properties. Careful consideration must also be given to the external environment in which a magnet must operate. The thermal, radiation, and oxidation environments in an accelerator must be taken into consideration during the design process. Permanent-magnet devices may also impact a vacuum environment, the quality of which is often critical for an accelerator.

The thermal environment impacts both mechanical and magnetic properties of permanent magnets and must be taken into consideration during the design process. Values for the thermal coefficients of remanence, $\alpha$, and coercivity, $\beta$, for all of the common rare-earth PM materials are quite large, varying from $\alpha \geq 3 \times 10^{-4} \mathrm{C}^{-1}$ and $\beta \geq 1 \times 10^{-3}{ }^{\circ} \mathrm{C}^{-1}$ for Sm.Co, and $\alpha \geq 1 \times 10^{-3}{ }^{\circ} C^{-1}$ and $\beta \geq 5 \times 10^{-3}{ }^{\circ} C^{-1}$ for Nd. $\mathrm{Fe}-\mathrm{B}$. Variation of remanence and coercivity within the "safeoperating range" for a given material, however, for rare earth PM materials is completely reversible once the PM has been properly heat treated[79]. Stable operation of PM devices for accelerator applications, even with the large thermal coefficients, is feasible if the thermal environment of the magnet material is carefully controlled.

Radiation in and around accelerators is usually copius and degrades the performance (remanence and coercivity) of PM materials. Radiation damage should be considered irreversible since it is not practical for in situ annealing or recharging of permanent magnets in an accelerator. The primary exposure to ionizing radiation for drift-tube PMQs and those outside beam pipes or vacuum vessels for most accelerators will be neutrons and photons ( $\boldsymbol{\gamma}$ - and $\mathrm{X}$-rays). Charged particles below $\sim 100 \mathrm{MeV} /$ nucleon kinetic energy are not likely to have sufficient energy to pass through the wall of a drift tube or beam pipe. The effective thickness of the drift-tube wall for an ion impinging at $2.5^{\circ}$ is $23 \mathrm{~mm}$ for a $1 \mathrm{~mm}$ physical wall thickness[56]. Procons, for instance, with less than 150 . $\mathrm{MeV}$ kinetic energy can not penetrate $23 \mathrm{~mm}$ wall thickness. All particles impinging on the drift-tube wall, regardless of energy, will interact with the drift-tube material, nominally copper, producing primarily $X$-rays at lower kinetic energies, and at higher energies, nuclear reactions will produce numerous products including copius $\gamma$-rays and neutrons.

Understanding which PM materials are most resistant to damage by radiation and what conditions effect that resistance is crucial to properly design magnets for modern highintensity accelerators. The first detailed study of radiation effects in PM materials[25] examined the effects of broad spectrum (reactor core) neutrons on Alnico and barium fertite. Other studies[76-78] have shown that PM remanence degrades far more rapidly with neutron dose than an equivalent $\gamma$-ray dose. Hence, studies at LANL have focused on radiation damage of PM materials induced by neutrons and investigated correlations between PM radiation damage and environmental parameters such as temperature and external fields, $H_{\|}$. Our data[70,76] show that the radiation resistance of PM matertals is correlated with the material type, material microcrystaline structure, the external magnetic field, and the energy product of the material. There is also some conflicting evidence that radiation resistance is correlated with sample temperature during irradiation $[70,80]$. The correlation between material type and susceptibility to radiation damage is evident in Fig. 6, where the rate of remanence loss for Nd-Fe-B materials is universally faster as a function of neutron fluence than for either Sm-Co material by as much as an order of magnitude.

One can expect significant $H_{\|}$opposing the magnetization vector of the PM material in some regions of a segmented 


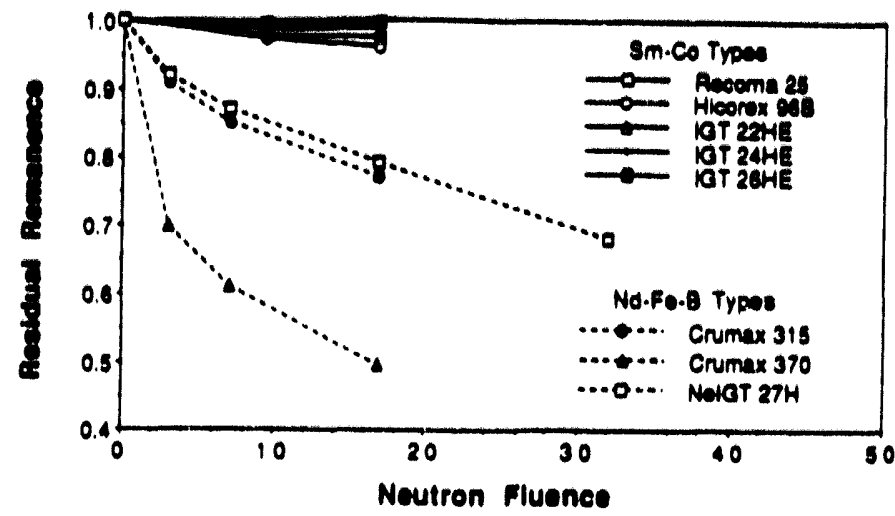

Fig. 6. Residual remanence, plotted as a function of total neutron fluence for samples of SmCos (Recoma 25 and Hicorex 96B), $\mathrm{Sm}_{2} \mathrm{Co} 17$ (ICT 22HE, 24HE, and 26HE), and $\mathrm{Nd}_{2} \mathrm{Fe}_{14} \mathrm{~B}$ (Crumax 315 and 370 and NeIGT 27H). 1 unit $=1 \times 10^{15} \mathrm{n} / \mathrm{cm}^{2}$. neutron fluence.

REC magnet[70]. Figure 7 shows the effect $H_{11}=-5.5 \mathrm{kG}$ (opposing the easy-axis orientation) has on the rate of remanence loss for two SmCos samples. These data show two extremes of the observed correlation between $H_{n}$ and residual remanence as a function of neutron fluence for $\mathrm{Sm}$-Co materials. Residual remanence is the post-irradiation remanence of a sample divided by the pre-irradiation remanence. The Recoma 25 sample showed no additional loss of remanence (with $-1 \%$ error bar) caused by $H_{n}=-5.5 \mathrm{kG}$ while the Hicorex 96B sample was severely degraded in the presence of the same $H_{n}$.

All SmCos and $\mathrm{Sm}_{2} \mathrm{Co}_{17}$ materials irradiated in our studies with no applied external field have lost less than 2.0\% of the initial remanence after exposure to $5 \times 10^{16} \mathrm{n} / \mathrm{cm}^{2}$. The rates of remanence loss for most samples imadiated in a large $H_{n}$ increased dramatically. Figure 8 shows data for eight different Sm-Co materials irradiated at $-80 \mathrm{~K}$ with

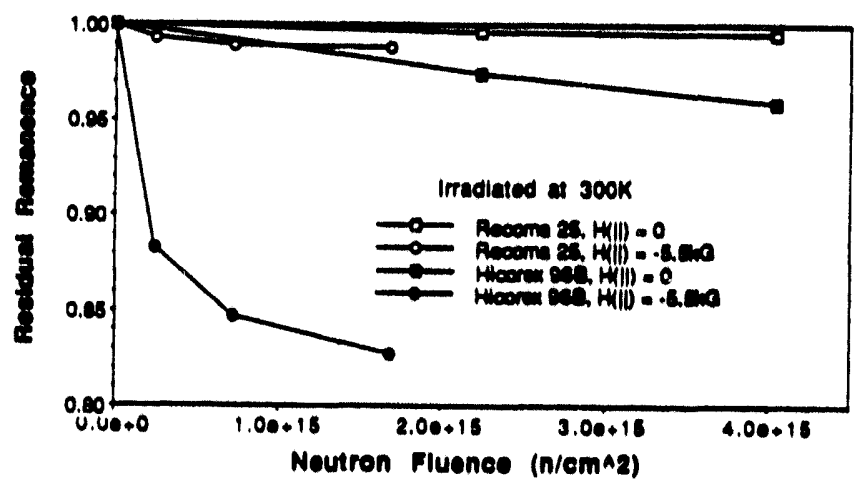

Fig. 7. Residual remanence, plotted as a function of total neutron fluence for samples of SmCo5. The loss of remanence for samples irradiated with $H_{\|}=0 \mathrm{kG}$ is compared with samples irradiated with $H_{\|}=-5.5 \mathrm{kO}$.
$H_{1}=-5.5 \mathrm{kO}$. A notable feature of these data is that increased remanence loss appears correlated with greater initial remanence for a given manufecturer.

Irradiation of PM samples has recently been complete at LAMPF accelerator at LANL where samples were exposed to spallation neutron fluences up to $10^{19}$ to $10^{20} \mathrm{n} / \mathrm{cm}^{2}$. Residual remanence for each sample will be measured after allowing the induced radioectivity of the samples to decay sufficiently for handling. Studies of radiation damage of PM materials are continuing (in collaboration with colleagues in the Materials Division at Los Alamos and colleagues at Camegie Mellon Research Institute) to better understand the mechanisms involved, and provide information to improve the radiation resistance of future PM materials.

The most reactive and easily oxidized PM materials commonly available today are the rare earth-iron-boron compounds of which $\mathrm{Nd}_{2} \mathrm{Fo} 14 \mathrm{~B}$ is most common. Exposure of a freshly ground or cut surface of Nd-Fe-B to air will result in surface oxidation within a few minutes. Cooling water is used extensively throughout all accelerator facilities, and PMQ magnets used in drift tubes, may be mounted inside the cooling channel for the drift tube and exposed directly to the cooling water. We found that a Nd-Fe-B permanent magnet formed a thick oxide layer when submerged in a sample of $>I M \Omega$ cooling water for a few weeks. Although these are very-qualitative experiments, they do serve to highlight the need to carofully isolate $\mathrm{Nd} 2 \mathrm{~F}{ }_{14} \mathrm{~B}$ from potentially oxidizing or hydrogen-containing environments with one of a variety of corrosion resistant coatings or potting in a suitable epoxy.

Moderate to high vacuum is required in the beam pipes through which charged particles are uransported. Outgassing from any materials exposed to vacuum must be compatible with the level of vacuum needed and materials required for ultrahigh-vacuum application ( $<10^{-9}$ torr) must be compatible with bake-out temperatures in excess of $200^{\circ} \mathrm{C}$. We have found that Sm-Co materials have outgassing rates similar to stainless steel, thus they are compatible with most typical vacuum requirements (>10-9 torr). Finally, devices placed in vacuum must be carefully designed to avoid "virtual leaks"

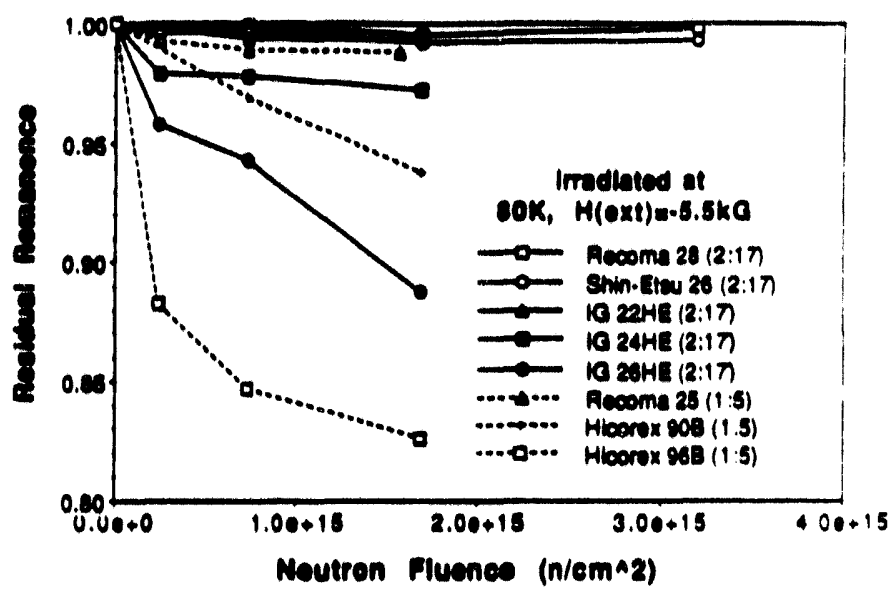

Fig. 8. Residual remanence, plotted as a function of total neutron fluence for samples of $\mathrm{SmCos}$ and $\mathrm{Sm}_{2} \mathrm{Co}_{17}$. Samples were maintained at $80 \mathrm{~K}$ and a field of $H_{\|}=-5.5 \mathrm{kG}$ was applied to all samples during irradiation. 
(pockets of trapped gas that have constricted paths to the vacuum). A typical segmented multipole configuration has a significant quantity of trapped air between the segments, potentially causing a virtual leak for a substantial period of time. This can be avoided by potting the magnet in a vacuum-compatible epoxy or building in pump-out paths. We have found it useful to consult with engineers familiar with vacuum technology when designing magnets that will be used in these applications.

\section{SUMMARY}

A wide variety of applications that PM materials have found in accelerators and associated devices have been described. Although designs and applications of PM systems from the mid 1950's to the early 1970's were often innovative, interest in these applications during this time period was sporadic. Consistent and significant interest in the use of permanent magnets for accelerator applications began in the mid 1970's when the recognition of the benefits of PM materials and the introduction of REC materials revitalized their use for accelerator applications. Early permanent-magnet designs relied heavily on experimentation and empirical methods. Today we have a vast array of tools with which to design devices utilizing PM materials from analytic formulas to two-dimensional and three-dimensional modeling codes. One can use these tools to design ideal magnets with ideal materials or model "real world" magnets assuming various fabrication and material imperfections. The real quality of a magnel, however, is determined by measuring the field and ultimately by the performance in the accelerator.

The largest application of PM materials for modern accelerators is in drift-tube quadrupole magnets. The most common PMQ design for modern DTL applicatious is the segmented REC magnet. These magnets generate the highest gradient in the smallest package and are compatible with drifttube structures. The design proposed by Skachkov[38] can potentially compete with the segmented REC magnets for use in DTL structures provided a sufficiendy high quality external field is used during the manufacturing process. Increasing the accelerating gradient by reducing the accelerating period or increasing the accelerator drive frequency, results in shorter and smaller drift tubes into which the PMQ magnets must fit. Couplo this with the increased focusing strength needed to compensate for space-charge forces in beans of ever increasing brightness and one can clearly see the trend toward a need for stronger PM materials. The materials used for these applications need to exhibit increased $B_{r}$ as well as $H_{c}$ and constant $\mu$ over the entire operating range of the material. Advances in the strength and quality of PM materials will positively impact future accelerator developments.

The trend toward increasing beam quality in new accelerators results in decreasing beam loss if all other parameters were hold constant. This trend in beam quality is often accompanied by a requirement for vastly increased beam intensity that results in a net increase in beam loss and the associated radiation dose. Heavy-ion fusion and high-energy colliding-beam accelerators will require final-focus magnets with very-high gradients and tolerance to significant radiation doses. Drift-tube PMQ life expectancies for high intensity linac projects currently under study at Los Alamos must be at leass several years, thus we are pursuing a research effort with the goal of devising new manufacturing processes to increase PM material resistance to radiation through the expansion of our understanding the mechanisms responsible for the radiation damage.

\section{REFERENCES}

[1.] E. Rutherford, Phil. Mag. 37581 (1919).

[2.] E. Rutherford, Proc. Roy. Soc. 117300 (London, 1927).

[3.] O. Ising, Archiv f. Matematik, Astronomi och Fysik 18 I (1924).

[4.] R. Wideroc, Archiv f. Elekt. 21387 (1928),

[5.] L. Alvarez, Phys. Rev. 701815 (1946).

(6.) L. Alvarez, et al., Rev. Sti. Inst. 26111 (1955).

[7.] E. D. Courant, M. S. Livingston, and H. S. Snyder, Phys. Rev. 881190 (1952).

[8.] J. P. Bleweth, Phys. Rev. 881197 (1952).

[9.] P. M. Lapostolle and A. L. Septier, Linear Accelerators (Wiley, New York, 1970).

[10.] B. Cork and E. Sajec, Phys. Rev. 92853 (1953).

[11.] F. B. Shull, C. E. MacFarland, and M. M. Bretscher, Rev. Sci. Inst. 25364 (1954).

[12.] H. A. Enge, Rev. Sci. Inst. 29885 (1958).

[13.] E. Regensteif, CERN 60-26, CERN PS Division intemal report, 1959.

[14.] P. M. Lapostolle, Los Alamos National Laboratory report LA-11601-MS (July 1989).

[15.] Muluple papers in Conf. on Lin. Accel. for High Energies, Brookhaven National Laboratory, April 1961 , and Conference on Lin. Accel. for High Energies, Brookhaven National Laboratory, August 1962.

[16.] A. M. Clogston and H. Heffner, J. Appl. Phys. 25 436 (1954).

[17.] W. F. Stubbins, Rev. Scl. Inst. 236666 (1956).

[18.] J. M. Wilcox, University of Califomia Radiation Laboratory report UCRL-3184 (1955).

[19.] R. Keller, et al., CERN report CERN-60-2 (1960).

[20.] J. W. Jagger and P. J. Riley, Rev. Sci. Inst. 38 955 (1967).

[21.] A. Harvey, et al., Proc. 1984 LINAC Conf., GSI.8411,14 (1984).

[22.] H. R. M. Hyder, et al., Intl. Conf. Elec. Accel. 352 (1973).

[23.] P. Thieberger and H. E. Wegner, Nucl. Inst. Meth. 122205 (1974).

[24.] J. N. Bradbury, E. A. Knapp, and D. E. Nagle, IEEE Trans. Nucl. Sci. NS.22 1755 (1975).

[25.] E. D. Bush, Proc. 1976 Proton Linac Conf. 363 (1976).

[26.] I. M. Kapchinskii, et al., Proc. 1976 Proton Linac Conf. 350 (1976).

[27.] B. P. Murin, et al., Prib. Tekh. Eksp. 1944 (USSR, 1976).

[28.] D. D. Mishin and R. M. Grechishkin, Sixth Intl. Conf. Mag. Tech., MT-6, 992 (1977).

[29.] I. M. Kapchinskii, et al., Prib. Tekh. Eksp. 51273 (USSR, 1977).

[30.] E. A. Swenson, et al., Proc. Tenth Intl. Conf. High Energy Accel. 29 (1977). 
[31.] V. I. Ilyushchenko and Y. V. Kulikov, Int. Inst. Nicl. Res. Rep. 13-10510 (1977).

[32.] N. Saito, et al., Proc. 3rd Intl. Wksp. REC Perm. Mag. Appl. 295 (1978) and Los Alamos National Laboratory report LA-7232-MS.

[33.] N. V. Lazarov and V. S. Skachkov, Proc. 1979 Proton Linac Conf. 380 (1979).

[34.] S. W. Herb, Proc. 1987 IEEE Part. Accel. Conf. 1434 (1987) and Nucl. Inst. Meth. A2s8 358 (1987).

[35.] J. P. Bleweth, Brookhaven National Laboratory repon AADD-89 (1965).

[36.] K. Halbach, Proc. 1979 IEEE Part. Accel. Conf. NS.26 3882 (1979).

[37.] R. F. Holsinger, Proc. 1979 Lin. Accel. Conf. 373 (1979).

[38.] V. S. Skachkov, A. V. Selin, I. M. Kapchinskiy, and N. V. Lazarev, Proc. 3rd Europ. Part. Accel. Conf. . 2 , 1400 (1992);V. Skachkov, USSR patent No. 1109031, Bulletin No. 30, 1984; and V. Skachkov, private communication (1992).

[39.] R. L. Gluckstem and R. F. Holsinger, Nucl. Inst. Meth. 187119 (1981).

[40.] R. L. Oluckstem and R. F. Holsinger, Proc. 1983 IEEE Part. Accel. Conf. NS.30 3623 (1983).

[41.] A. F. Zeller, et. al., IEEE Trans. Magn. 24990 (1988).

[42.] D.B. Barlow, R.H. Kraus, Jr., R.P. Martinez, and R. Meyer, Thirteenth Intl. Conf. Mag. Tech., MT-13, (these proceedinge, 1993).

[43.] R. H. Kraus and B. Campbell, 1990 NPB Tech. Symp. (1990) and Los Alamos National Laboratory document LA-CP.90-349 (1990).

[44.] K. Halbach, et al., Proc. 1985 IEEE Part. Accel. Conf. NS.32 3643 (1985).

[45.] K. Halbach, Proc. 1986 Lin. Accel. Conf. 407 (1986).

[46.] B. Feinbers, et al., Proc 1989 IEE Part. Accel. Conf. 375 (1989) and B. Feinberg, ot al., Proc. 1988 Lin. Accel. Conf. (1988).

[47.] D.B. Barlow, R.H. Kraus, Jr., and R.E. Meyer, Thirteenth Intl. Conf. Mag. Tech., MT.13, (these proceedings, 1993).; R. H. Kraus, Jr., to be published in Nucl. Inst. Meth. ; K. C. D. Chan, R. H. Kraus, J. Ledford, K. L. Meier, R.E. Meyer, D., Nguyen, R. L. Sheffield, F. L. Sigler, L. M. Young, T.-S. Wang, W. L. Wilson, and R. L. Wood, Nucl. Inst. Meth. , A318, 148 (1991); and R. L. Sheffield, R. H. Austin, K. C. D. Chan, S. M. Gierman, J. Kinross-Wright, S. N. Kong, D. C. Nguyen, S. J. Russell, and C. A. Timmer, Proc. 1993 Part. Accel. Conf., Washington, DC, (IEEE Pub., in press)

[48.] J. Arianer and R. Geller, Amn. Rev. Nucl. Sci. 31 19 (1981).

[49.] R. Geller, Ann. Rev. Nucl. Sci. 4015 (1990).

[50.] K., Halbach, Proc. 1986 Lin. Accel. Conf. 407 (1986).

[51.] C. Geisik and R. R. Stevens, Proc. 1991 NPB Tech. Symp. (1991) and Los Alamos National Laboratory document LA-CP-91-301 (1991).

[52.] E. W. McCune, Froc. 1987 IEEE Part. Accel. Conf. 370 (1989).

[53.] J. D. Bowman and R. H. Heffner, Nuci. Inst. Meth. 148503 (1978).
[54.] R. H. Kraus, et al., Nucl. Inst. Meth. A264 327 (1988).

[55.] J. J. Croat, et al., Appl. Phys. Lelt. 44148 (1984), and M. Sagawa, et al., J. Appl. Phys. 552083 (1984).

[56.] S. O. Schriber, Los Alamos National Laboratory document LA.UR-88-3169, presented at 1988 Part Accel. Conf., Chicago (unpublished), and S. O. Schriber and R. A. Hardekopf, Los Alamos National Laboratory report LA.12022.PR (1991).

[57.) R. H. Kraus, et a., Proc. 1991 NPB Tech. Symp. (1991) and Los AlamoaNational Laboratory document LA.CP.91-275 (1991).

[58.] D. Liska and R. H. Kraus, Proc. 1988 Lin. Accel. Conf. (1988).

[59.] H. Wollnik, Nucl. Inst. Meth. 103479 (1972), and O. W. Orime and F.Wath, Beam Optics of Quadrupole Probe-Forming Systems (Adam Hilger, Ltd., Bristol, England, 1984).

[60.] M. Baltay, et al., Proc. 1987 IEEE Part Accel. Conf. 1431 (1987).

[61.] W. Davies-White, A. Hution, and A. Harvey, IEEE Trans. Mag. 241327 (1988).

[62.] J. E. Spancer, Proc. 1985 IEEE Part. Accel. Conf. NS.32 3666 (1985).

[63.] K. Egawa and R. M. Taylor, Proc. 1989 IEEE Parl. Accel. Conf. 360 (1989).

[64.] J. Spencer and H. Stuckd, Proc. 1989 IEEE Part. Accel. Conf. 360 (1989).

[65.] J. Chavanne, J. LaForest, and R. Pauthenet, Mat. Res, Soc. Symp. Proc. 962307 (1987).

[66.] M. Kumada, et al., Proc. 9th Intl. Conf. Mag. Tech. (MT.9) 142 (1985).

[67.] Y. Iwashita, et al., Proc. 1990 Lin. Accel. Conf. 746 (1990).

[68.] S. O. Sankar and F. Pourarian, private comm. (1990)

[69.] E. B. Boltich and W. E. Walace, Solid State Comm. 53529 (9185).

[70.] R.H. Kraus, Jr., Permanen Magnets and Their Applications, S.G. Sankar, Ed. (World Scientific, London, 1993, in press)

[71.] M. T. Menzol and H. K. Stokes, Los Alamos National Laboratory documents LA-UR-87-115 (1987) and L.A. UR-87-126 (1987).

[72.] Magsoft Corp. (Troy, New York, copyright 1987).

[73.] Vector Fields, Lid. (Oxford, England, copyright 1991)

[74.] K. Halbach, Nucl. Inst. Meth. 169 1 (1980); K. Halbach, Nucl. Inst. Meth. 187 109; (1981); K. Halbach, Nucl. Inst. Meth. 198213 (1982).

[75.] R. L. Gluckstern and R. F. Holsinger, Proc. 1981 Lin. Accel. Conf. 214 (1981).

[76.] E. W. Blackmore, Proc. 1985 IEEE Part. Accel. Conf. NS-32 3669 (1985).

[77.] J. Cost, of al., Mat. Res, Soc. Symp. Proc. 96321 (1987).

[78.] H. Spitzer and A. Weller, KFA.Julich report SNQ I N/BH 22-05-84 (1984).

[79.] F. Coninckx, ot al., CERN/SPS report TIS-RP/IR/8307 (1983).

[80.] O..P. Kahkonen, S. Makinen, M. Talvitie, and $M$. Manninen, University of Jyvaskyla preprint JYFL 20/91 (1991). 

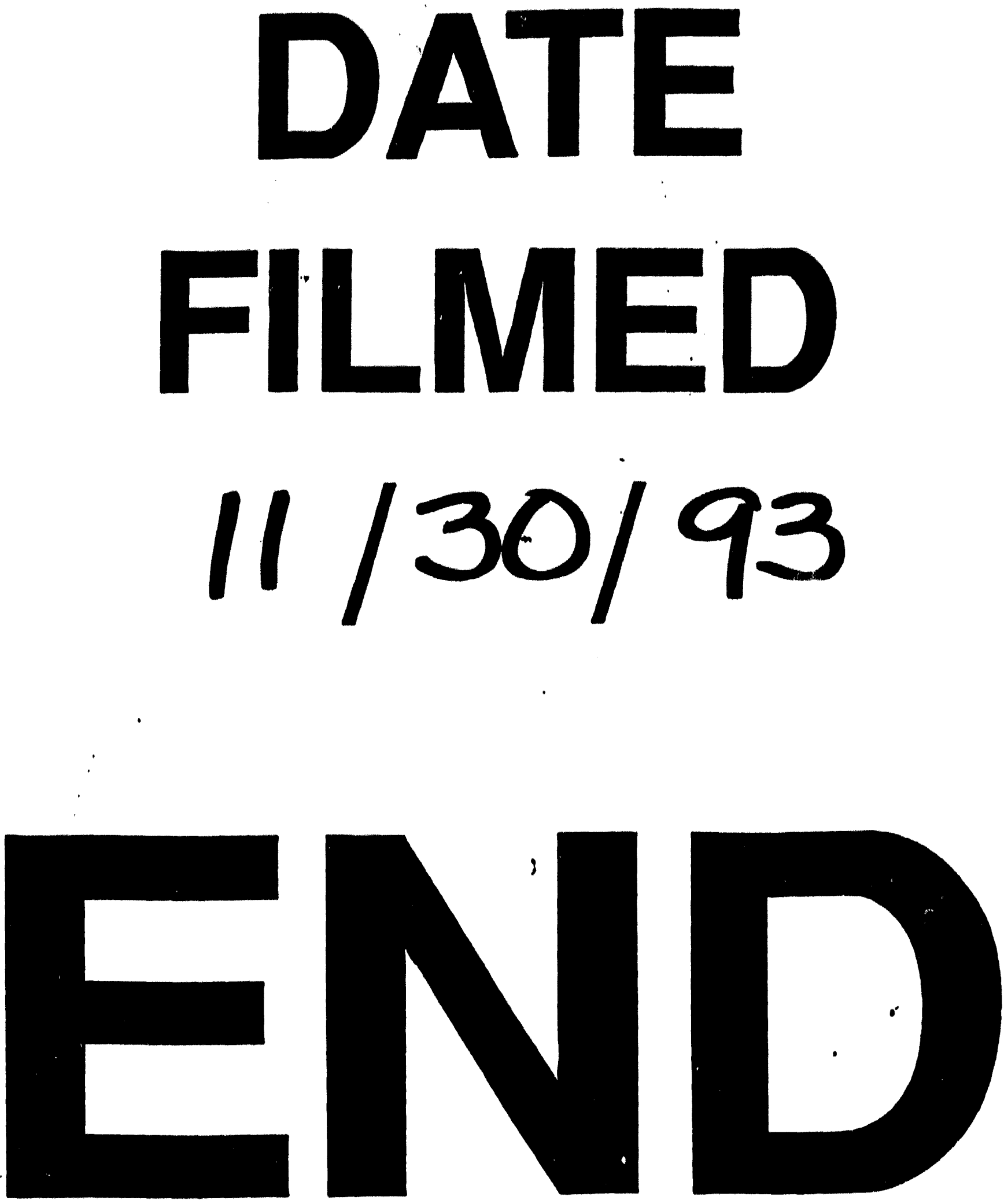

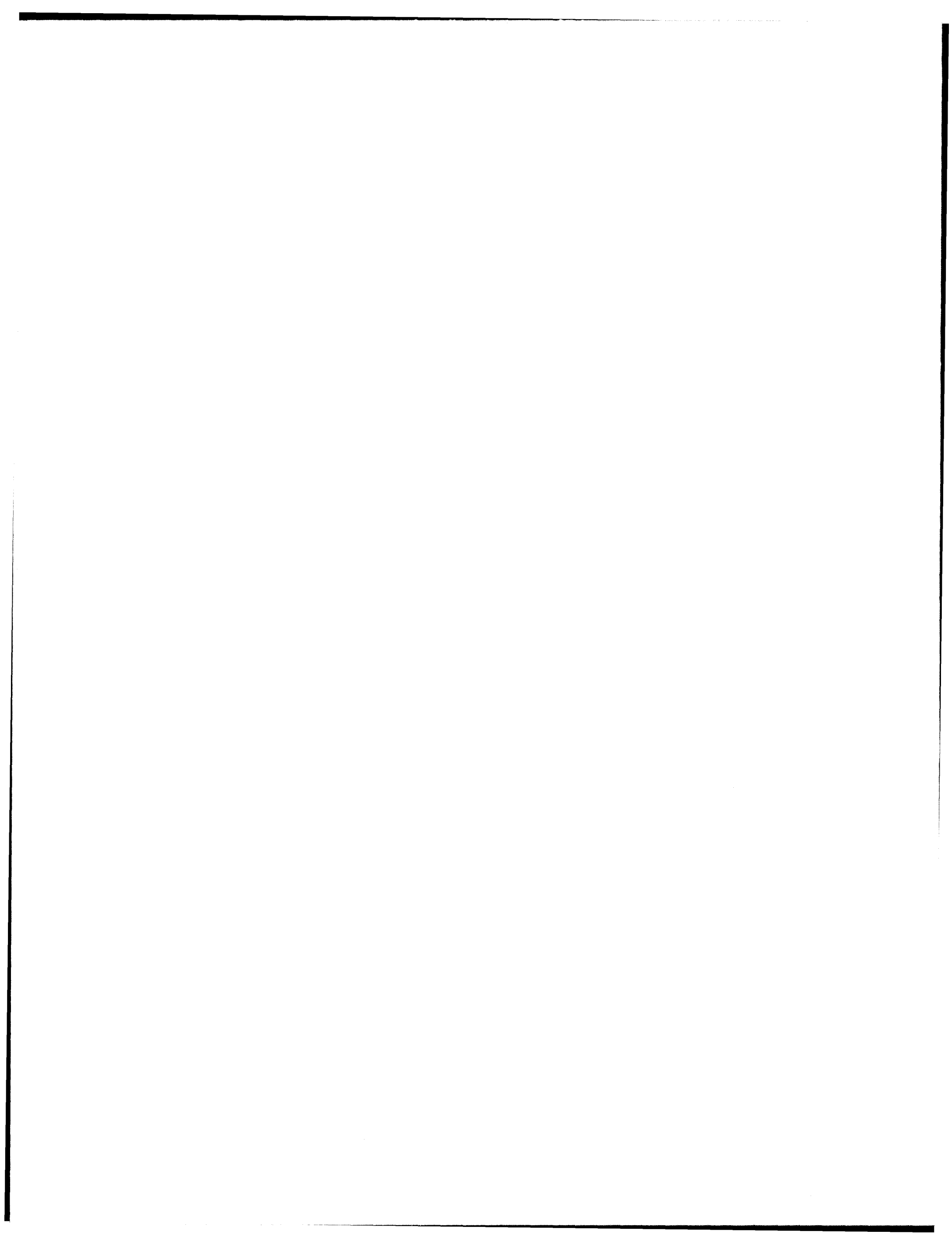